\title{
La maternidad a edades tempranas en Mauritania: una perspectiva geodemográfica
}

\section{Early childbearing in Mauritania: a geodemographic perspective}

\author{
Josefina Domínguez-Mujica,* Mercedes de los Ángeles \\ Rodríguez-Rodríguez* y Laudy Rivero-Rodríguez** \\ *Universidad de Las Palmas de Gran Canaria, España \\ **Servicio Canario de Salud, España
}

\begin{abstract}
Resumen
Los embarazos a edades tempranas constituyen un grave problema social y afectan al desarrollo de las adolescentes, a su salud y a la sobrevivencia de sus hijos e hijas. Existe un fuerte vínculo entre la pobreza y el embarazo a temprana edad, pues éste es más frecuente entre mujeres jóvenes pobres. Un análisis detallado de este fenómeno en Mauritania permite apreciar notables diferencias de carácter geográfico en cuanto a estas pautas reproductivas en el seno del país. Por ello, este artículo se ha trazado el objetivo de indagar en los factores geodemográficos y sociodemográficos que condicionan la maternidad a edades tempranas en Mauritania, para ofrecer algunas reflexiones acerca de las circunstancias que se han de tener en cuenta para desarrollar programas de intervención que reduzcan su incidencia.
\end{abstract}

Palabras clave: Mauritania, embarazos tempranos, fecundidad, pobreza.

Abstract

The early childbearing constitute a serious social problem and affect the development of adolescents, their health and the survival of their sons and daughters. There is a strong linkage between poverty and pregnancy at an early age, as this is more frequent among poor young women. A detailed analysis of this phenomenon in Mauritania allows us to perceive notable geographical differences related with these reproductive patterns within the country. Therefore this paper aims to inquire into the geo-demographic and socio-demographic factors conditioning the early childbearing in Mauritania, to provide some reflections regarding the environments that must be taken into account to develop intervention programs that reduce their incidence.

Key words: Mauritania, early pregnancy, fertility, poverty. 


\section{INTRODUCCIÓN}

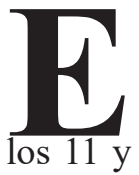

1 embarazo en adolescentes se ha definido como el embarazo de niñas y jóvenes de 15-19 años (OMS, 2018), aunque la propia

OMS define "la adolescencia como la etapa que transcurre entre los 11 y 19 años, considerándose dos fases, la adolescencia temprana 11 a 14 años y la adolescencia tardía 15 a 19 años, por lo que los embarazos en menores de 19 años son embarazos adolescentes o embarazos a edades tempranas.

Este hecho constituye un grave problema social y afecta al desarrollo de las adolescentes, a su salud y a la sobrevivencia de sus hijos e hijas. En lo que respecta a África, se trata de una realidad que se ha de analizar a la luz de las recomendaciones de organismos internacionales que defienden la necesidad de demorar la reproducción de las jóvenes hasta la plenitud de su desarrollo, principios recogidos, entre otros documentos, en la Carta Africana sobre los Derechos y el Bienestar del Niño.

Existe un fuerte vínculo entre la pobreza y la maternidad temprana, pues ésta es más frecuente entre mujeres jóvenes de menores recursos. Por ende, cuando se estudia la maternidad temprana, se reconoce, de inmediato, su importancia en los países en desarrollo, así como la del contexto propio de cada uno de ellos. Esta mirada geográfica obliga a describir las variaciones individuales y contextuales de los embarazos de adolescentes para sugerir las necesarias intervenciones (Gausman et al., 2019), máxime si se tiene en cuenta que, como señalaba Mabinge Ngom, Director Regional de la Oficina de África Occidental y Central del Fondo de Población de Naciones Unidas:

las jóvenes y mujeres juegan un papel inconmensurable en la construcción de los principios del mundo del mañana, y cabe esperar cambios en las normas sociales y valores que las guían... Aprovechar el dividendo demográfico es una oportunidad que África no debe dejar pasar (UNFPA, 2016: 3).

Nuestro estudio trata de contribuir a este objetivo, indagando en los factores geodemográficos y socio-demográficos que condicionan la maternidad a edades tempranas en Mauritania, para ofrecer algunas reflexiones acerca de las circunstancias que se han de tener en cuenta para desarrollar programas que reduzcan la incidencia de los embarazos tempranos. 
La maternidad a edades tempranas en Mauritania: una perspectiva geodemográfica / J. DOMINGUEZ MUJICA et al.

\section{ANTECEDENTES, OBJETIVOS E HIPÓTESIS DE LA INVESTIGACIÓN}

La investigación acerca de la maternidad a edades tempranas tiene una gran tradición en un amplio espectro de disciplinas, no sólo en aquellas vinculadas a la esfera médico-sanitaria (Obstetricia y Ginecología, Salud Pública, Salud Reproductiva, Medicina Familiar, Salud Materna, Salud de la Infancia y Adolescencia, Planificación Familiar, etcétera.) sino también en otras del ámbito de las Ciencias Sociales, especialmente en la Demografía, la Sociología, la Geografía de la Salud o la Geografía Social y Cultural. Por ello, los encuentros científicos y las publicaciones en los que se aborda este tema nos permiten reconocer la atención que brindan los centros de investigación y las revistas científicas a una cuestión que se mantiene como una prioridad de la salud, porque condiciona el futuro de las mujeres y del conjunto de la sociedad en muchos países. Así lo demuestra el considerable número de estudios realizados en el seno de organismos como la Organización Mundial de la Salud (OMS), Population Council, United Nations Population Fund (UNFPA), United Nations International Children's Emergency Fund (UNICEF), Centro Latinoamericano y Caribeño de Demografía (CELADE) de la Comisión Económica para América Latina y el Caribe de Naciones Unidas (CEPAL), etcétera.

Además de las recomendaciones que contienen estos estudios, en ellos se aborda cómo influyen los contextos locales en la maternidad a edades tempranas, así como las consecuencias de los embarazos en niñas y adolescentes, especialmente en los países en desarrollo. De hecho, como ya apuntamos, se da un gran paralelismo entre el menor nivel de desarrollo de los países y regiones de la Tierra y estos embarazos. Como señalaron Ganchimeg et al. (2014: 40) en nombre de OMS, "se estima que 11 por ciento de nacimientos en todo el mundo los proporcionan mujeres jóvenes adolescentes entre 15 y 19 años, y que más de 90 por ciento de estos nacimientos ocurren en países con nivel de ingresos bajo y medio". En consecuencia, muchas de las investigaciones tratan de encontrar comportamientos comunes entre ciertos países, afrontando una perspectiva de conjunto, o bien seleccionan algunos de ellos en función de la importancia de la variable analizada.

A modo de ejemplo, en el primer caso se hallan las publicaciones de Singh, 1998; Santhya, 2011; Neal et al., 2012; Chandra-Mouli et al., 2013; Svanemyr et al., 2015; Santelli et al., 2017; etcétera. En el segundo, en el contexto de América Latina, encontramos tanto visiones de conjunto (CELADE, 2004; Chackiel, 2004) como trabajos específicos como el de 
Menkes y Suárez, 2003 y el de Stern, 2004 sobre México; León et al., 2008 sobre Chile; Alarcón Argota, 2009 sobre Cuba; Binstock y Näslund-Hadley, 2013 sobre Paraguay; Mendoza y Subiría, 2013 sobre Perú; Lemon et al., 2017 sobre Guatemala; Shakya et al., 2019 sobre Honduras; etcétera., entre otros.

Para el ámbito geográfico del continente asiático, en algunas publicaciones se analiza la maternidad a edades tempranas en ciertas áreas como el Sudeste asiático (Acharya et al., 2010 o Kennedy et al., 2011) o bien en ciertos países: Tailandia (Thaithae y Thato, 2011); Bangladesh (Nahar y Min, 2008); Emiratos Árabes Unidos (Green and Smith, 2007), etcétera.

Con respecto al continente africano, sucede otro tanto; hay tanto investigaciones en relación con grandes áreas regionales: África Subsahariana (Maswikwa et al., 2015; Hindin, 2014; Lloyd and Mensch, 2008), como acerca de países concretos: Camerún (Calvès, 2000); Madagascar (Almanza and Sahn, 2018); Kenia (Were, 2007), etcétera.

También encontramos estudios relativos a los embarazos a edades tempranas en los países desarrollados en su conjunto (Singh and Darroch, 2000) así como en ciertos países en los que el foco de atención se dirige a grupos sociales desfavorecidos, especialmente en Estados Unidos de América (Barcelos, 2014; Penman-Aguilar, 2013). En dichos estudios, sus autores ofrecen una reflexión crítica y un estado de la cuestión, identificando los factores socioeconómicos que influyen en la maternidad a edades tempranas: desempleo, bajos niveles de ingresos, bajos niveles educativos, desventajas del ámbito de convivencia, trastornos físicos en el ámbito de convivencia, desigualdad de ingresos a nivel del vecindario y bajo estatus socioeconómico.

En relación con los países desarrollados, también se analizan estos embarazos en ciertas minorías étnicas o de inmigrantes, tanto en Estados Unidos como en Europa en su conjunto (Sobotka, 2008). En estas investigaciones se pone de manifiesto el diferenciado comportamiento de estas minorías, aunque a medida que pasa el tiempo, estos grupos tienden a adaptarse al comportamiento de la mayoría de los residentes en estos países (Lundström and Andersson, 2012).

En este contexto, la maternidad a edades temprana en el caso de Mauritania, objeto de este estudio, ofrece un gran interés. En primer lugar, porque los embarazos de niñas y adolescentes en Mauritania, aunque han sido foco de atención específica por diferentes organizaciones internacionales, no lo han sido del mundo académico, por lo que existen muchas lagunas al respecto. En segundo lugar, porque los factores de orden cultural y so- 
cioeconómico ganan todo su valor interpretativo en este caso y, finalmente, porque la dimensión geográfica revela no sólo pautas de comportamiento diferenciadas en el seno del país, sino también algunas claves que han de orientar las políticas sociales, especialmente las educativas y las de salud reproductiva.

En consecuencia, nos hemos marcado los siguientes objetivos en este estudio. Primeramente, cuantificar y caracterizar el fenómeno de la maternidad a edades tempranas en Mauritania; en segundo término, establecer las relaciones oportunas entre los niveles socioeconómicos y educativos de la población y los embarazos a edades tempranas, desde una perspectiva geográfica. También analizaremos el impacto que ha tenido la implementación de los programas socio-sanitarios, en cuanto a la atención a los partos, lo que nos permite contextualizar estos resultados con una reflexión sobre las iniciativas más exitosas para prevenir y retrasar el embarazo a edades tempranas.

Estos objetivos nos permiten formular la siguiente hipótesis, en correspondencia con el estado actual del conocimiento en esta materia: la relación que guardan los embarazos a edades tempranas en Mauritania, con las diferencias de carácter socioeconómico y cultural que caracterizan el país, obliga a diseñar estrategias específicas de acuerdo con una mirada espacial, pues la geografía importa. Es decir, habrá que considerar el carácter nómada, rural o urbano de las poblaciones y los resultados previos de los programas educativos y de salud implementados en los distintos ámbitos geográficos del país, para contrarrestar las prácticas reproductivas que descansan en principios culturales y socioeconómicos que afectan al desarrollo de las niñas y jóvenes, a su salud, a la sobrevivencia de sus hijos e hijas, así como a su capacidad de libre elección ante la descendencia.

\section{FUENTES Y METODOLOGÍA DE ESTUdIO}

Para aproximarnos a la hipótesis de investigación anteriormente descrita, desarrollamos un análisis cuantitativo, a partir de distintas fuentes estadísticas, y un estudio cualitativo, que se valió de una entrevista semiestructurada a informantes cualificados. En su conjunto, la combinación del análisis estadístico con los resultados de la entrevista nos permitió alcanzar los objetivos que nos trazamos en este estudio.

Los datos acopiados para el análisis cuantitativo tienen como unidades de análisis geográfico las wilayas de Mauritania (regiones). Dichos datos proceden de diferentes fuentes, entre ellas la Multiple Indicator Cluster Survey, en su acrónimo MICS, realizada por UNICEF. 
Como es sabido, desde su concepción en 1995, MICS se ha convertido en la mayor fuente de datos estadísticos, comparables internacionalmente, en relación con las mujeres y los niños de todo el mundo y continuará siendo una fuente de información decisiva para la Agenda de Desarrollo Sostenible de 2030, que ha de medir los indicadores de los Objetivos del Desarrollo Sostenible (ODS). La última MICS realizada en Mauritania correspondió a la quinta ronda, y por ello su sigla es MICS 5. Concretamente, utilizamos de ella los indicadores relativos a: porcentaje de mujeres de 15 a 19 años que han comenzado su vida fértil, porcentaje de mujeres de 15 a 19 años que han tenido un parto con un nacido vivo antes de los 15 años, porcentaje de mujeres de 20 a 24 años que han tenido un parto con un nacido vivo antes de los 18 años, porcentaje de mujeres de 15 a 49 años que han tenido un parto atendido por personal cualificado en los dos últimos años (en el momento de la realización de la encuesta MICS5) y porcentaje de salas de parto en buen estado.

También fueron utilizados los datos de la Enquête Permanente sur les Conditions de Vie des ménages 2014, en sus siglas, EPCV, realizada por la Office National de la Statistique - République Islamique de Mauritanie (acrónimo ONS-RIM). Esta encuesta es la sexta de su tipo que se realiza en el país. Se llevó a cabo enteramente con recursos propios y con la asistencia técnica del Observatorio Económico y Social de Estadísticas del África Subsahariana (AFRISTAT) en cuanto al tratamiento de datos y de su cómputo. Su informe puso de relieve las tendencias del fenómeno de la pobreza en Mauritania en 2014. De dicha encuesta se obtuvo información acerca de la incidencia de la pobreza por wilayas (porcentaje de individuos que viven por debajo del umbral de pobreza, umbral predeterminado en 2014 para el país ${ }^{1}$ ), tasa de alfabetización para la población femenina mayor de 15 años, y tasa neta de escolarización ${ }^{2}$ de las mujeres en el nivel de enseñanza secundario (15-24 años).

La información sociosanitaria, por otra parte, fue obtenida de la Carte Sanitaire Nacionale de la Mauritanie (Ministère de la Santé, RIM, 2014) proyecto desarrollado por Agencia Española de Cooperación Internacional para el Desarrollo (AECID) y el Ministerio de Salud mauritano, del 20122014, y por documentos del Plan National de Developpement Sanitaire, Période 2017-2020, (Fase II, Volumne 1 y 4) del Ministère de la Santé de la RIM, disponibles todos on-line.

\footnotetext{
1 Para Mauritania (2014) se consideraron pobres a las personas que vivían con menos de 169.445 MRO (ex ouguiyas) al año, es decir, menos de $1.30 \$$ diario.

${ }^{2}$ La tasa neta de escolarización es la relación porcentual entre el alumnado de la edad considerada respecto al total de población de esa edad.
} 
Además, se recopilaron datos procedentes del Recensement Général de la Population et de l'Habitat (RGPH, 2013) (ONS-RIM): cifras de población por wilayas, cifras de población según medios geográficos de residencia (nómada, rural y urbano) y estructura por sexo y edad de la población.

A todos estos datos primarios se sumaron otros, procedentes de algunos informes específicos acerca de las mujeres, realizados ad hoc, como el de Investir dans l'égalité hommes femmes pour la transformation de l'Afrique, del Groupe de la Banque Africaine de Développement (2015), o que derivan de la información del propio Censo, como el Rapport de l'Etude du Genre à partir des données du RGPH (ONS, 2016). Con todas estas fuentes de información, entre otras, se calcularon las tasas de fecundidad por edades, el índice sintético de fecundidad ${ }^{3}$ y los cocientes de localización de las jóvenes de entre 15 y 19 años. También se realizaron análisis bivariados entre, por una parte, las variables: tasa de alfabetización de mujeres de más de 15 años; tasa neta escolarización femenina en el nivel secundario (15-24 años); población rural; incidencia de la pobreza; y parto atendido por personal cualificado; y, por otra, las tasas de fecundidad por grupos de edad $(<15 ; 15-19 ; 20-24)$. Para ello establecimos cálculos de niveles de correlación en los que se demostrara una significación menor al alfa fijado (95 por ciento de confianza y alfa de 0.05 ), adoptándose la fórmula de Spearman, pues sirve para correlacionar variables aleatorias, tanto continuas como discretas.

Tras la prueba de hipótesis, procedimos a hacer un análisis clúster que se mostró en un dendograma, lo que nos permitió clasificar la muestra en un número pequeño de grupos muy similares entre sí y muy disimilares del resto. Se tomó la medida de distancia cuadrática euclidiana y el método de agrupamiento por centroide (medida de tendencia central de las variables del grupo).

Finalmente, georreferenciamos los datos de población urbana, rural y nómada, fecundidad, escolarización y atención sanitaria, según las estructuras mayores de la división político-administrativa del país, las wilayas o regiones (13 en el momento del estudio y hoy 15). También georreferenciamos la información de la población al nivel de las 55 moughataas (estructura equivalente a provincias) y de las 207 comunas (estructura equivalente a municipios); dentro de las comunas las localidades se agrupan en localidades de más de 500 habitantes, de entre 200 y 500 y menos de 200 habitantes. Los resultados se expresaron cartográficamente mediante

\footnotetext{
3 Número de hijos que tendría una mujer al final de su vida fecunda, si durante la misma su comportamiento correspondiese, en cada edad, con el de la serie de tasas específicas de fecundidad por edad.
} 
un mapa de coropletas a nivel de wilayas y uno de círculos graduados a nivel de comunas y moughataas (poblamiento), ambos elaborados a partir de ArcGIS.

En cuanto a las entrevistas realizadas, nos dirigimos a informantes cualificados del ámbito de la salud, concretamente a una Doctora Pediatra, Directora General de la Escuela Nacional de Salud Pública de Mauritania, Zeinebou Haidy Haimoudane y a un consultor español de la Fundación Estatal, Salud, Infancia y Bienestar Social (acrónimo FCSAI), Santiago Alonso Pardo, que tuvo una participación activa en la elaboración del mapa sanitario de Mauritania. Entrevistamos también a la responsable de la oficina Child Protection de UNICEF Mauritania, Fatma Soueid Ahmed; a una cooperante española, May San Alberto, que trabajó como enfermera en Mauritania y, además, realizó algunos proyectos fotográficos relacionados con la vida cotidiana de mujeres mauritanas; a una galerista y artista mauritana del ámbito de la pintura, Ami Sow, que ha desarrollado su arte para sensibilizar a los espectadores en la necesidad de defender a las jóvenes mauritanas; y a la representante mauritana de la Organización no Gubernamental Alliance citoyenne, Atikatou Dieng, una organización creada en 2016, para luchar, entre otros cometidos, contra el matrimonio infantil. A todos ellos se les entregó un cuestionario semiestructurado. Las respuestas a dicho cuestionario fueron tabuladas, tras definir las distintas categorías de análisis, al mismo tiempo que fueron seleccionadas algunas afirmaciones significativas, como complemento al trabajo cuantitativo desarrollado.

\section{ANÁLISIS DE RESULTADOS: LOS INDICADORES DE FECUNDIDAD}

El análisis de los embarazos en adolescentes mauritanas requiere de una atención específica a los indicadores de fecundidad. En primer lugar, el índice sintético de fecundidad arroja una cifra muy elevada para el conjunto de Mauritania (4.3 hijos por mujer), al mismo tiempo que ofrece importantes diferencias atendiendo al carácter del medio, registrándose los más altos niveles en las poblaciones nómada y rural (Figura 1).

Figura 1: Índice sintético de fecundidad según ámbitos geográficos

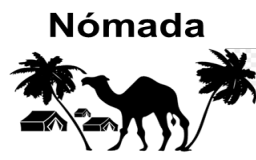

4,7

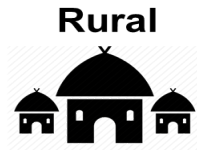

4,6

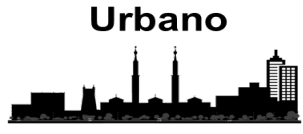

4,0

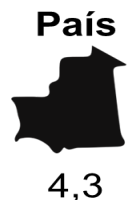

4,3

Fuente: elaboración propia con base en Recensement Général de la Population et de l'Habitat, 2013. 
Por otra parte, el número de nacimientos por grupos de edad de las mujeres refleja no sólo su elevada proporción, sino también la temprana incorporación de éstas a la vida fértil, aunque con diferencias notables según el medio, como reflejan la Tabla 1 y la Figura 2, pues hay una mayor proporción de mujeres que comienzan su vida fértil antes de los 15 años en las wilayas con mayores porcentajes de poblaciones nómada y rural, como demuestra esta correlación $(\mathrm{R}=0.69 ; \mathrm{p}<0.01)$.

Tabla 1: Proporción de mujeres con vida fértil antes de los 15 años y de las poblaciones nómada y rural

\begin{tabular}{lcc}
\hline & $\begin{array}{c}\text { Mujeres con vida fértil antes } \\
\text { de los 15 años (\%) }\end{array}$ & $\begin{array}{c}\text { Población nómada } \\
\text { y rural (\%) }\end{array}$ \\
\hline Hodh Echargui & 6.1 & 81.3 \\
Hodh El Gharbi & 5.6 & 84.6 \\
Assaba & 6.1 & 72.9 \\
Gorgol & 5.9 & 71.3 \\
Brakna & 4.0 & 77.2 \\
Trarza & 2.9 & 74.4 \\
Adrar & 5.7 & 48.5 \\
Nouadhibou & 2.9 & 4.5 \\
Tagant & 3.4 & 83.1 \\
Guidimakha & 4.6 & 70.3 \\
Tiris Zemmour & 0.7 & 7.0 \\
Inchiri & 0 & 35.0 \\
Nouakchott & 1.5 & 0 \\
\hline
\end{tabular}

Fuente: elaboración propia con base en MICS5, 2015 y Recensement Général de la Population et de l'Habitat, 2013. 


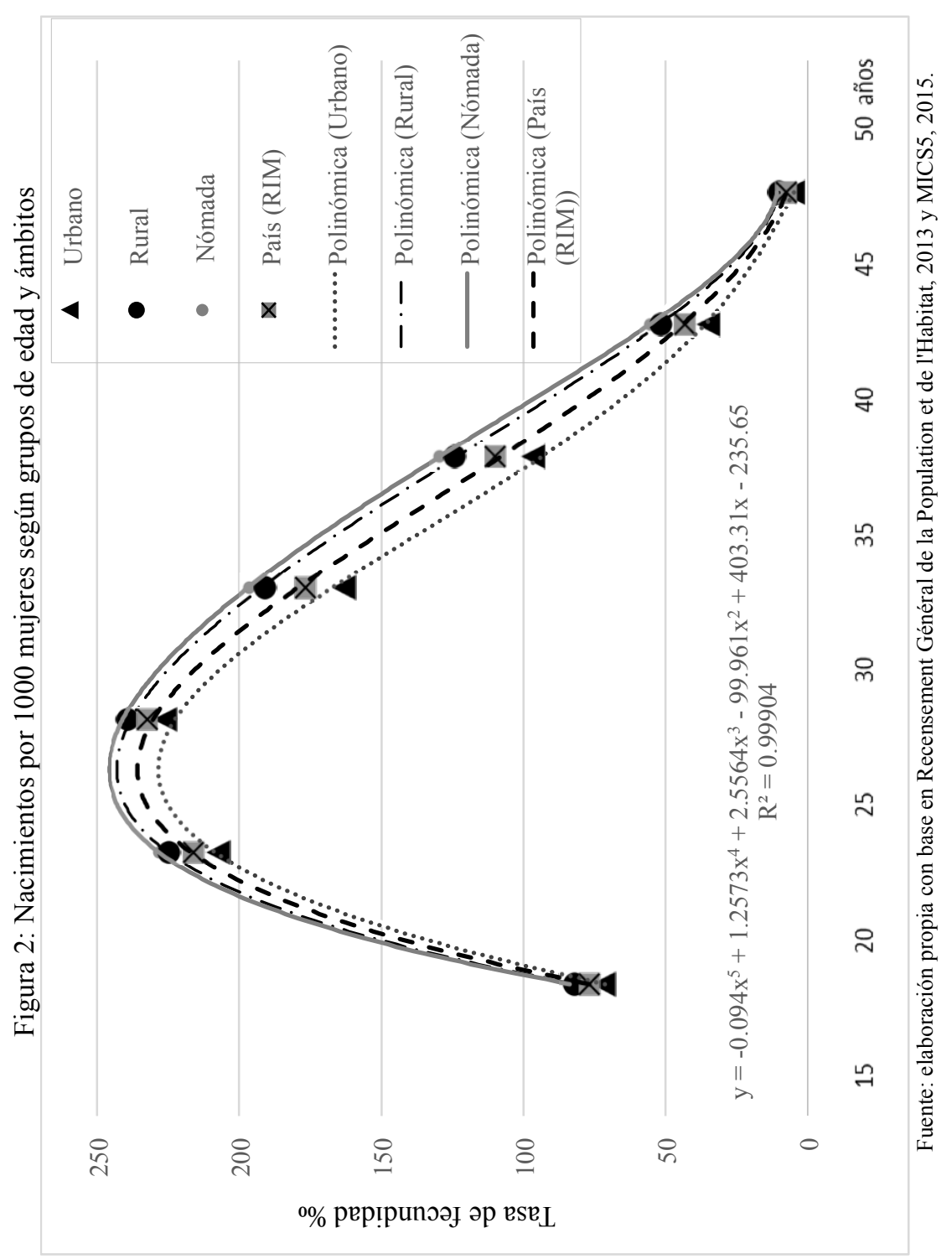


La maternidad a edades tempranas en Mauritania: una perspectiva geodemográfica / J. DOMINGUEZ MUJICA et al.

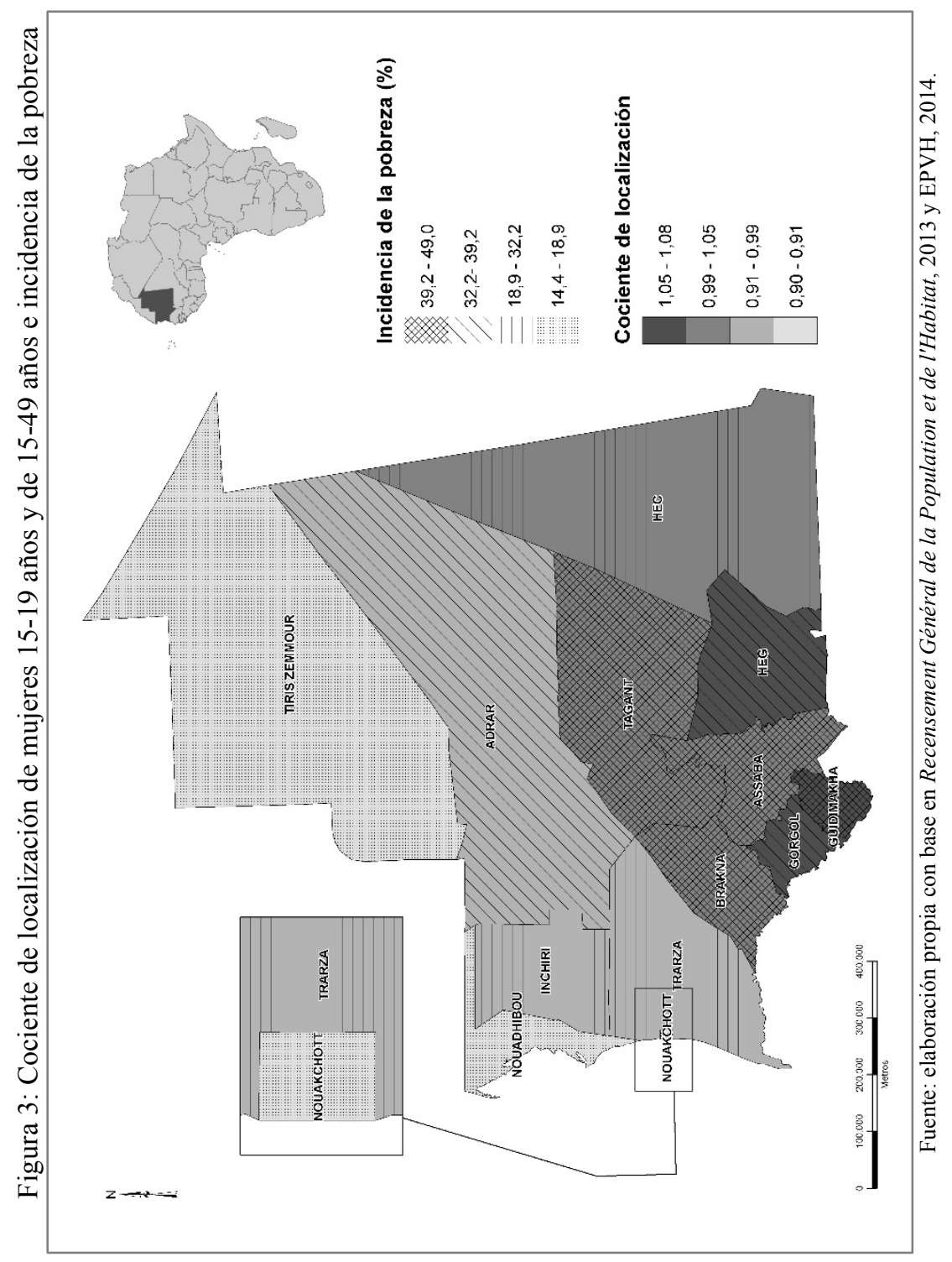


Desde una perspectiva geográfica, el cociente de localización de las mujeres en edad fértil y la incidencia de la pobreza quedan reflejados en el mapa de la Figura 3. En él se puede apreciar que la mayor proporción de mujeres en edad fértil se halla en las wilayas de Hodh Echargui, Hodh El Gharbi, Assaba, Gorgol, Adrar y Guidimakha, lo que apunta a las zonas en las que se han de redoblar los esfuerzos de formación de las mujeres en salud reproductiva, máxime si se tiene en cuenta que dichas wilayas arrojan altos niveles de población que vive bajo el umbral de pobreza, especialmente las de Hodh El Gharbi, Assaba, Gorgol, Adrar y Guidimakha. En términos territoriales, es el sur-sureste del país el que se halla más necesitado del desarrollo de estas iniciativas.

Por tanto, no cabe duda de la íntima relación que guardan los niveles socioeconómicos de la población con las diferencias de comportamiento ante la reproducción. Se cumple así la idea defendida, entre otros autores, por Walker (2012), cuando afirmaba que, en África, los países con las tasas más elevadas de matrimonios tempranos son también los países con las más altas tasas de pobreza. En este caso, cambiamos la escala de análisis para confirmar que los mayores niveles de pobreza, en relación con el predominio de las poblaciones nómada y rural, arrojan las mayores tasas de fecundidad en edades tempranas. A esta consideración geográfica se une la expresada por el consultor en salud entrevistado, Alonso, quien apunta también a la mayor prevalencia de embarazos tempranos en el extrarradio de las ciudades, especialmente de Nouakchott. Estas circunstancias no se deben olvidar cuando se intenta afrontar intervenciones activas para combatir el problema de la elevada incidencia de los matrimonios y la maternidad a edades tempranas.

\section{LOS FACTORES CULTURALES}

En las entrevistas en profundidad desarrolladas se reveló la importancia de una serie de circunstancias propias de la mentalidad y la costumbre, que más allá de las evidencias del trabajo cuantitativo, informan de la posición de subordinación de la mujer y de las dificultades para erradicar ciertas prácticas sociales que les impiden su autonomía y, en consecuencia, su capacidad de demorar su reproducción hasta la plenitud de su desarrollo. Entre ellas, por su mayor importancia en relación con los embarazos a edades tempranas, cabe mencionar los matrimonios de niñas y las circunstancias que los favorecen, así como ciertas costumbres como las mutilaciones genitales y el engorde, que ponen en peligro el bienestar, la salud y la capacidad de desarrollo de las mujeres. Dado que las mutilaciones genitales han 
cosechado una gran atención en el ámbito de la salud y de la literatura de género, y que tratar este asunto desbordaría los objetivos de este artículo, vamos a centrarnos en los matrimonios tempranos, en las causas que los originan, así como en una de las costumbres que, aunque ha ido perdiendo importancia en los últimos años, ha sido poco estudiada en el ámbito académico, la del engorde de la mujer soltera, una práctica que se lleva a cabo para incrementar su valor en el 'mercado matrimonial'.

\section{El matrimonio infantil}

La práctica tradicional de los matrimonios infantiles persiste en todo el mundo y tiene efectos negativos sobre las jóvenes que se casan tempranamente y sobre su descendencia, así como sobre el conjunto de sus comunidades y sociedades. Conviene recordar, en este sentido, que en Mauritania los embarazos se producen en el seno del matrimonio excepto en casos muy aislados. Por ello es importante reconocer que, de acuerdo con el estudio realizado por la Ford Foundation (2013), cinco países de África occidental habían involucionado hacia cifras más elevadas de matrimonio temprano con el paso de los años (Cabo Verde, Togo, Burkina Faso, Benin y Mauritania), habiéndose producido, en el caso de Mauritania, un incremento de 0.7 por ciento en cuanto a las niñas casadas de 15 a 19 años entre 2000-2005 y 2006-2011 (desde 24 a 24.7 por ciento), una cifra que, con todo, es más baja que la que proporcionaron el propio Ministerio de Asuntos Sociales de Mauritania y World Vision, con motivo del Día Internacional de la Niña, celebrado el 11 de octubre de 2018 en Nouakchott (37 por ciento de niñas casadas antes de los 18 años, según datos aportados en el evento).

La información más reciente acerca de la relación entre los embarazos de adolescentes y el matrimonio temprano los proporcionaron Wodon et al. (2019), en su estudio. En él calcularon la proporción de nacimiento infantil temprano (early child birth) atribuido a la práctica del matrimonio temprano, usando datos de Encuestas Demográficas y de Salud. En el caso de Mauritania, la estimación intermedia fue de 90 por ciento por niños, ${ }^{4}$ una cifra notablemente alta, superior a la media calculada para los 26 países para los que se llevó a cabo el estudio, que era de 84.4 por ciento.

Los factores relacionados con el embarazo temprano han sido identificados en estas categorías: tasas de finalización de la escuela primaria y secundaria de mujeres; residencia urbana / rural; tasa de participación femenina en la fuerza laboral y nivel de ingresos, aunque las relaciones entre

${ }^{4}$ El nacimiento infantil temprano (early child birth) se define como la proporción de hijos nacidos de una madre de menos de 18 años de edad (\%). 
estas variables y la temprana edad en el matrimonio son complejas. Así lo pone de manifiesto el hecho de que un ligero incremento del nivel de escolarización femenina, de la tasa de urbanización y de la tasa de actividad femenina en Mauritania, en los periodos anteriormente señalados, no ha venido acompañado de una notable reducción de los matrimonios tempranos, pues ciertas inercias socio-culturales condicionan su persistencia. No obstante, como analizaremos más adelante, sí que se advierte una estrecha correlación entre la menor prevalencia de matrimonios y embarazos en adolescentes en aquellas áreas geográficas del país con un mayor nivel de formación femenina, con un predominio de la población urbana frente a la rural y nómada y en las que se han implementado programas de salud. Por ello, cuando se le preguntaba a la galerista Sow acerca de los factores que contribuían al matrimonio precoz, ella respondía que "la pobreza era el factor número uno".

De forma específica, en cuanto a los condicionantes socioculturales, algunos autores han señalado que en el mercado matrimonial de las sociedades patriarcales, el honor de una familia está fuertemente relacionado con la pureza de sus miembros femeninos (Moghadam, 2004) y que las novias mayores, que han podido adquirir experiencia fuera del hogar paterno pueden crear incertidumbre con respecto a su 'pureza' y, por lo tanto, tienen peor reputación y menores opciones que las novias más jóvenes (Wahhaj, 2015). Como señala Soueid Ahmed en la entrevista, "lo que influye en el matrimonio temprano es colocar a la niña en un lugar seguro, es decir, a salvo de relaciones fuera del matrimonio".

En relación con los matrimonios en las sociedades preindustriales de Eurasia, otros apuntan a que las novias jóvenes tienen más probabilidades de ser obedientes o dóciles y aceptar más fácilmente las reglas y formas del nuevo hogar (Goody, 1990). De hecho, cuanto más joven es la edad de la novia, menos posibilidades hay de que se manifieste con respecto a la elección del cónyuge o de que se le dé pie a que exprese su consentimiento para casarse.

A estas circunstancias se puede agregar un factor económico, la riqueza o dote de la novia, es decir, la transferencia de dinero, bienes o servicios de la familia de la novia a la del novio, lo que hace que los padres tomen todas las decisiones con respecto a los acuerdos matrimoniales de sus hijas. La dote es un factor que desvaloriza a las mujeres, exacerbando su marginación y explotación, particularmente porque mercantiliza o monetiza el valor de las niñas (Amin y Bajracharya, 2011) y favorece el matrimonio precoz entre familias pobres porque, "cuanta mayor es la dote, más atrae a 
las familias de menores recursos", en palabras de la entrevistada Atikatou Dieng. No obstante, según Soueid Ahmed, otra de las entrevistadas, en los matrimonios tempranos prevalecen lazos de solidaridad de los miembros del grupo antes que la dote, pues según la religión islámica, "el novio tan sólo ha de aportar un cuarto de dinar para los preparativos de la boda".

Por otra parte, la práctica del matrimonio a temprana edad no puede olvidar su relación con la poliginia. El estatus de las mujeres en uniones matrimoniales formales o informales de carácter polígamo se ve afectado no sólo por la posición de la mujer con respecto a su esposo sino también por su posición con respecto a las otras co-esposas. En consecuencia, lo más habitual es que la nueva esposa sea más joven que las anteriores, como señala el entrevistado Alonso: "la tendencia por parte de los hombres polígamos es la de ir abandonando a las mujeres de más edad para sustituirlas por otras más jóvenes, incluso adolescentes". Como señala Tertilt (2005: 1,341) "las mujeres en países poligínicos se casan, en promedio, 5.1 años antes y tienen 2.2 hijos más que las mujeres en países monógamos" y así parecen demostrarlo sus datos, pues según sus cálculos en Mauritania había 11.2 por ciento de mujeres casadas en una unión polígama (tanto en matrimonios legales como en otras formas de convivencia) en la fecha de su estudio. En palabras de la cooperante entrevistada, San Alberto: "los hombres quieren de nuevo mujeres jóvenes y si no les está permitida la poligamia, se divorcian para casarse otra vez".

En otro orden de cosas, 60 por ciento de las muertes maternas en Mauritania se produce entre niñas de 12 a 15 años, según declaraciones de Aïssata Bâ (Ministerio de Asuntos Sociales de Mauritania y World Vision, 2018). Es decir, una edad más temprana en el matrimonio tiene efectos negativos en la autonomía y bienestar de las mujeres y en su salud reproductiva. En consecuencia, poner fin al matrimonio infantil también es uno de los objetivos del Gobierno mauritano, que ya en el año 2001 había fijado la edad legal de matrimonio en los 18 años.

Para lograr que se respete esta norma, el propio gobierno ha recurrido a argumentos religiosos, como los defendidos por la Asociación de Ulemas de Mauritania, en palabras del imam Bouna Oumar Ly: "El Islam ha prohibido el matrimonio de las niñas antes de que maduren, pues el matrimonio implica un sentido de responsabilidad, que no se puede esperar de una niña" (Ministerio de Asuntos Sociales de Mauritania y World Vision, 2018). Sin embargo, según la entrevistada Dieng, "el matrimonio de Aicha con el Profeta a una edad temprana es un pretexto para desoír estas reco- 
mendaciones por parte de muchos creyentes" aunque, en palabras de Sow "la religión [ìslámica] nunca ha permitido la violencia de género".

Por tanto, a pesar de las indicaciones religiosas y del establecimiento de la edad legal, siguen produciéndose matrimonios tempranos. Además, el marco legal e institucional mauritano contiene brechas que aún no permiten alinear la normativa matrimonial con lo dispuesto por la Convención Internacional sobre los Derechos del Niño y otros instrumentos jurídicos supranacionales. Por ejemplo, según Soueid Ahmed, la ley sobre violencia de género no pudo promulgarse a pesar de su aprobación en el Consejo de Ministros y el Senado, porque no fue aprobada por la Comisión del Ministerio de Asuntos Religiosos, que evalúa la conformidad de las leyes con la Shari'a islámica.

Finalmente, erradicar el matrimonio infantil también se ha convertido en un hecho clave para lograr los Objetivos de Desarrollo Sostenible (ODS), tal y como defiende Girls not Brides (Niñas, no Novias), una asociación mundial de más de mil organizaciones de la sociedad civil, comprometidas a poner fin al matrimonio infantil y uniones tempranas, para permitir que las niñas y adolescentes desarrollen todo su potencial.

\section{El engorde de las jóvenes}

La alimentación forzada de las niñas y adolescentes en Mauritania es una de las prácticas tradicionales, discriminatorias y perjudiciales para la mujer, anclada en costumbres ancestrales (Agence Française de Développement, 2016) que, aunque ha ido perdiendo cada vez más importancia, se mantiene en ciertas zonas con una mayor población nómada y rural (ONS, 2016), especialmente en ámbitos donde predomina la población árabe. El objetivo de esta práctica es la de engordar a las jóvenes con una finalidad estética, dado que el canon de belleza para ciertos hombres mauritanos es el de mujeres obesas, un rasgo de fertilidad y de éxito económico que las habilita para casarse con aquellos que cuentan con un cierto patrimonio y recursos económicos. Como sucede en muchas otras poblaciones árabes nómadas, que luchan por sobrevivir en áridos desiertos, la gordura se aprecia como un signo de bienestar (Associated Press, 2007).

Rguibi y Belahsen en su estudio sobre las mujeres con obesidad y sobrepeso en Marruecos, plantearon que las "ideas culturales sobre lo que es deseable y atractivo tienen importantes implicaciones en el desarrollo de la imagen del cuerpo y pueden favorecer las prácticas que logran o mantienen esta particular imagen" (2006: 622). Así, en referencia a Mauritania, Hof utilizaba esta reveladora metáfora: "en Mauritania, la obesidad es tradicio- 
nalmente uno de los criterios de belleza por excelencia y a los hombres les gusta perderse entre los pliegues del cuerpo de sus esposas" (Hof, 2015).

Esta tradición origina problemas de salud (diabetes, afecciones cardíacas, problemas articulares, etcétera.) pues, para lograr el exceso de peso, en la cultura mauritana las niñas y adolescentes son obligadas por sus familias a comer en exceso, lo que debe interpretarse como un signo de violencia contra la mujer. La práctica del leblouh o gavage (palabra esta última de origen francés, referida al engorde de los gansos para obtener foie gras), comienza desde edades tempranas. Entre los 12 y 14 años y, a veces, incluso a los siete, las niñas son sometidas a una dieta calórica muy elevada, de hasta 16 mil calorías al día, para cumplir con los criterios de belleza anteriormente mencionados. Recientemente, incluso, se ha llegado a utilizar la cortisona o los productos para el engorde del ganado para reforzar el gavage, lo que implica riesgos añadidos para la salud (Agence Française de Développement, 2016). El objetivo es alimentarlas para hacer realidad el dicho maure (árabe-bereber) de que "la mujer ocupa la atención de su espacio", señalaba en 2019 Aminatu Mint El Moktar (El País, 2019), presidenta de la Asociación de Mujeres Cabeza de Familia y candidata para el Premio Nobel de la Paz en 2015, cuando denunciaba el engorde.

Las estadísticas sobre la magnitud de esta práctica son escasas, pues tiene muy poca visibilidad. Los informes de organismos nacionales mauritanos consideran a leblouh una forma de violencia femenina, tal como la mutilación genital, el matrimonio precoz, los abusos sexuales, la violencia doméstica y la marginación (ONS, 2016; Commission Nationale des Droits de l'Homme, 2017). Sin embargo, el marco jurídico del país carece de disposiciones específicas que penalicen a los padres y eviten los daños físicos y psicológicos relacionados con la alimentación forzada.

En 2008, un cuarto de la población femenina total en Mauritania había sufrido alimentación forzada (UNICEF, 2011). Sin embargo, según datos del Rapport de l'Etude du Genre à partir des données du RGPH, las cifras se elevan, pues fue víctima de estas prácticas 67 por ciento de las niñas antes de los 10 años, 45 por ciento antes de los ocho años y 18 por ciento antes de los seis. Además, se calcula que, en 42 por ciento de los casos, la alimentación forzada duró entre 12 meses y cuatro años, periodo en el que las niñas tenían que ingerir hasta 25 litros de leche de camella, una gran cantidad de comida y, al mismo tiempo, disminuir el ejercicio físico. La meta era un sobrepeso tal, que a los 8 años puedan pesar $85 \mathrm{~kg}$. y, fácilmente, más de 140 cuando lleguen a los 14 o 15 años (ONS, 2016). 
Para poner fin a estas brutales prácticas de alimentación, el gobierno lanzó a principios del presente siglo una campaña de televisión y radio que destacaba los importantes riegos que entrañaba el engorde para la salud de las jóvenes. A partir de 2004, además, ha ido desarrollando programas de concienciación en comunidades locales, con la participación de líderes religiosos y se han creado y puesto en marcha centros de escucha gestionados por ONG y una unidad técnica para luchar contra todas las formas de violencia femenina (Groupe de la Banque Africaine de Developpment, 2015). No obstante, se hace difícil erradicar estas prácticas porque descansan en la costumbre, en herencias culturales y en una concepción de sumisión de la mujer.

\section{LOS FACTORES GEO-DEMOGRÁFICOS Y SOCIOECONÓMICOS}

La población mauritana, con 3'535,368 habitantes y una densidad de 3.4 $\mathrm{hab} / \mathrm{km}^{2}$ según el Recensement Général de la Population et de l'Habitat (RGPH, 2013), presentaba una distribución geográfica muy desigual, atendiendo a las trece regiones administrativas del país (wilayas). Así, sus habitantes se concentraban en Nouakchott (la capital) (958,399 habitantes), en las wilayas del sur, limítrofes con Mali (Hodh Echargi y Hodh El Gharbi), con Senegal (Assaba), su río y afluentes (Guidiaka, Gorgol, Brakna y Trarza), así como en la wilaya del otro gran núcleo urbano del país, Nouakchott. El mapa de poblamiento de la Figura 4 pone de manifiesto estas diferencias en cuanto a la distribución de la población, en especial la dispersión de la población en pequeñas localidades, un factor de gran repercusión para el diseño de las políticas activas en materia educativa y sanitaria, que pueden retrotraer los niveles de embarazos a edades tempranas.

El hecho de que la concentración de población se produzca en áreas próximas al río, que se ven inundadas con frecuencia en la estación de lluvias y en zonas cercanas a la carretera principal (Figura 4), son factores añadidos, que han de ser valorados en las estrategias de dotación de infraestructura educativa y sanitaria y de la implementación de programas de formación. 
La maternidad a edades tempranas en Mauritania: una perspectiva geodemográfica / J. DOMÍNGUEZ MUJICA et al.

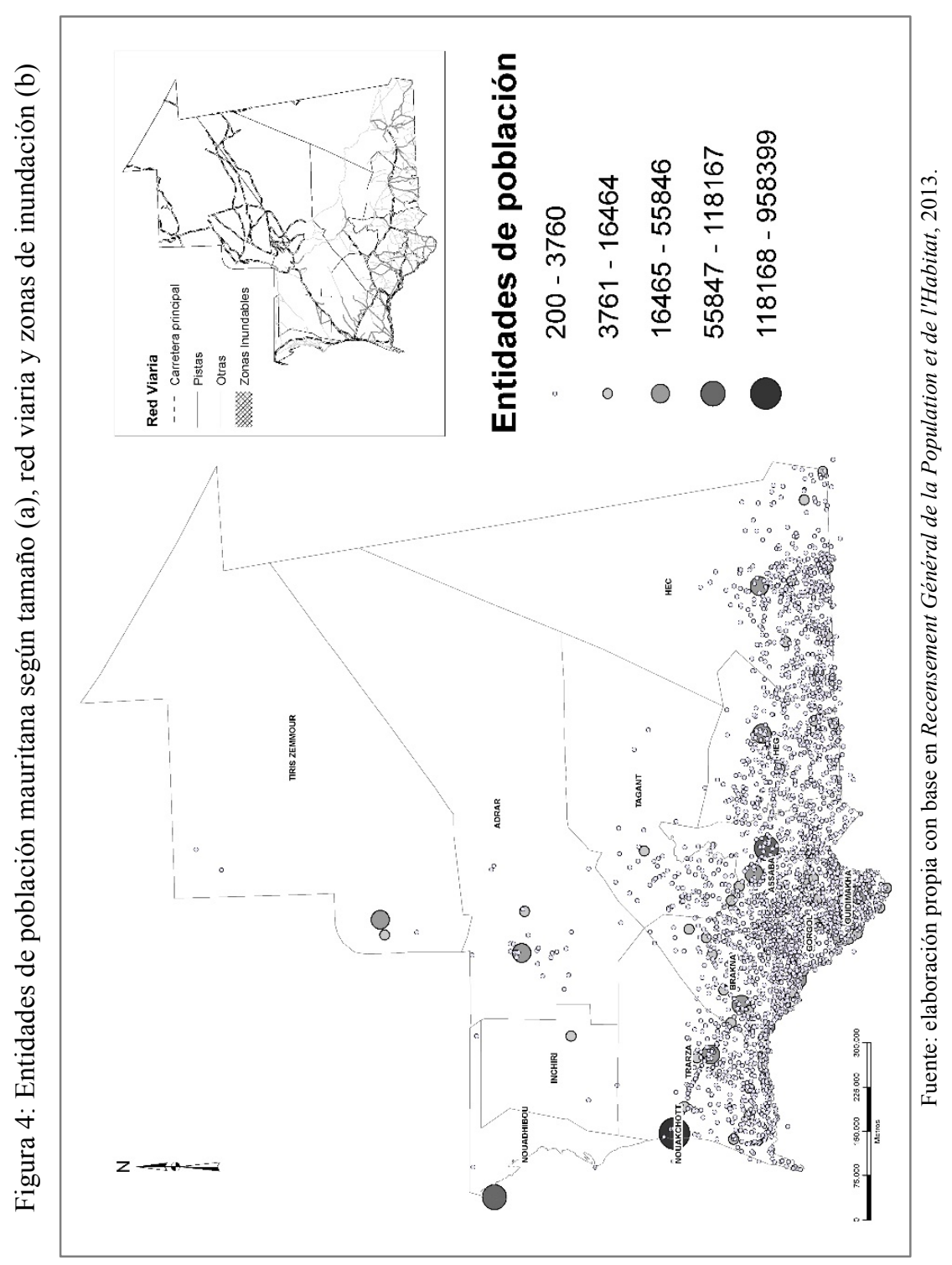


A este desequilibrio en la distribución de la población se suma el que, de acuerdo con la Enquête Permanente sur les Conditions de Vie des ménages 2014, de la Office National de la Statistique, se producen también grandes contrastes territoriales desde el punto de vista de la proporción de población que vive bajo el umbral de pobreza, como ya hemos comentado. Sin embargo, la comparación entre ambos indicadores no refleja correspondencia. Es decir, predomina la población que vive bajo el nivel de pobreza en zonas densamente habitadas.

Por una parte, hay zonas muy pobladas, como las grandes ciudades de Nouadhibou y de Nouakchott, en las que se registra una menor proporción de población pobre, aunque hay localidades, especialmente en sus periferias, donde se alcanzan también unos elevados niveles de pobreza. Por otra parte, a la inversa, en wilayas muy pobladas, se registran unos niveles de ingresos muy bajos, como sucede en Hodh Echargui, Hodh El Gharbi o Guidimakha, lugares en los que la escasa disponibilidad de recursos lleva a una menor movilidad de la población, especialmente, de la femenina. Es decir, que la atracción de las zonas más ricas del país no genera una intensidad migratoria tal que suponga una disminución de población de las zonas más pobres, aquellas próximas al río Senegal y a la carretera principal que une el país en su tramo sur, de oeste a este.

Estos factores son de una gran importancia para la planificación de la atención a la salud reproductiva de las mujeres, pues las intervenciones de los programas que se implementen han de considerar estos desequilibrios geográficos de población-riqueza. Ahora bien, si se combina el dato de la población bajo el umbral de pobreza, con el relativo a las poblaciones urbana, rural y nómada de las distintas wilayas, sí se confirman pautas comunes en cuanto al reparto de la pobreza, pues hay una estrecha correlación positiva $(\mathrm{R}=0.85 ; \mathrm{p}<0.01)$ entre las wilayas que tienen una mayor proporción de población pobre y aquéllas que cuentan con una ratio más elevada de población rural y nómada, como se puede apreciar Tabla 2.

Las wilayas con menor proporción de población bajo el umbral de la pobreza, además de las dos grandes entidades urbanas, son las de Inchiri, por su riqueza minera, las septentrionales de Tiris Zemmour y Adrar, al sur del Sahara, y la de Trarza, en el extremo suroccidental del país. Los niveles más altos de pobreza y de población rural se hallan en las wilayas meridionales y orientales, más alejadas de la costa. 
La maternidad a edades tempranas en Mauritania: una perspectiva geodemográfica / J. DOMÍNGUEZ MUJICA et al.

Tabla 2: Población bajo el umbral de pobreza según medios geográficos

\begin{tabular}{lrr}
\hline & Incidencia de pobreza $\%$ & Población rural y nómada $\%$ \\
\hline Hodh Echargui & 28.3 & 81.3 \\
Hodh El Gharbi & 39.2 & 84.6 \\
Assaba & 43.5 & 72.9 \\
Gorgol & 38.2 & 71.3 \\
Brakna & 43.3 & 77.2 \\
Trarza & 32.2 & 74.4 \\
Adrar & 36.9 & 48.5 \\
Nouadhibou & 14.8 & 4.5 \\
Tagant & 49.0 & 83.1 \\
Guidimakha & 49.1 & 70.3 \\
Tiris Zemmour & 18.9 & 7.0 \\
Inchiri & 23.7 & 35.0 \\
Nouakchott & 14.4 & 0 \\
\hline
\end{tabular}

Fuente: elaboración propia con base en EPVH, 2014 y Recensement Général de la Population et de l'Habitat, 2013.

\section{EDUCACIÓN Y ATENCIÓN SOCIO-SANITARIA}

Unos parámetros semejantes a los ya descritos en relación con la pobreza, se aprecian cuando analizamos indicadores educativos como la tasa de población femenina alfabeta de más de 15 años y la tasa de escolaridad de las jóvenes de entre 15 y 24 años que cursan estudios secundarios (Tabla 3).

De nuevo se confirma que las cifras más bajas de estos indicadores corresponden a las wilayas más pobres, de forma que hay una relación inversamente proporcional entre los mayores niveles educativos de niñas y mujeres y el predominio de poblaciones nómada y rural, así como de aquella que vive bajo el umbral de pobreza.

También resultan muy indicativos los datos de asistencia a la escuela secundaria según géneros, porque ponen de manifiesto que el acceso a la formación no sólo se ve dificultado por factores relacionados con la pobreza sino también con la propia consideración del papel de la mujer en la sociedad mauritana, como revela la Tabla 4. 
Tabla 3. Niveles de alfabetización femenina en Mauritania

\begin{tabular}{lrr}
\hline & $\begin{array}{r}\text { Proporción de mujeres } \\
\text { alfabetas }(>15 \text { años })(\%)\end{array}$ & $\begin{array}{r}\text { Proporción de mujeres } \\
\text { en secundaria (15-24 años }(\%)\end{array}$ \\
\hline Hodh Echargui & 65.4 & 12.3 \\
Hodh El Gharbi & 52.8 & 25.6 \\
Assaba & 64.4 & 18.5 \\
Gorgol & 44.6 & 22.9 \\
Brakna & 62.5 & 29.2 \\
Trarza & 81.6 & 37.2 \\
Adrar & 80.5 & 36.7 \\
Nouadhibou & 81.7 & 40.7 \\
Tagant & 73.9 & 21.7 \\
Guidimakha & 41.5 & 16.6 \\
Tiris Zemmour & 89.8 & 35.5 \\
Inchiri & 83.0 & 35.3 \\
Nouakchott & 81.9 & 42.3 \\
\hline
\end{tabular}

Fuente: elaboración propia con base en MICS 5, 2015.

Desde la perspectiva socio-sanitaria, nos hemos servido de uno de los indicadores que refleja la situación de la salud reproductiva. Nos referimos al de la proporción de partos atendidos por personal cualificado (con formación reglada en salud o parteras). Dicha variable ha sido la escogida para el análisis multivariante y su elección viene condicionada porque guarda una estrecha relación con la propia estructura geográfica de la prestación de los servicios de salud en Mauritania.

El sistema mauritano de salud está estructurado de forma piramidal, con tres niveles de actuación: el nivel operativo o primario (moughataa) con puestos de salud y centros de salud (634 y 105, respectivamente), que también cuenta con el apoyo de cientos de unidades básicas de salud, situadas en zonas con un radio superior a los 10 kilómetros de dichos puestos o centros. En segundo lugar, se halla el nivel intermedio, con dos tipos de hospitales: los situados en las moughataas más pobladas o aisladas y los hospitales regionales, llamados centros hospitalarios regionales $\mathrm{u}$ hospitales regionales. 
La maternidad a edades tempranas en Mauritania: una perspectiva geodemográfica / J. DOMINGUEZ MUJICA et al.

Tabla 4: Niveles de asistencia a la escuela secundaria según géneros en Mauritania

\begin{tabular}{lcc}
\hline & $\begin{array}{c}\text { Niños } \\
\text { Tasa neta de asistencia a la } \\
\text { escuela secundaria (ajustada) }\end{array}$ & $\begin{array}{c}\text { Niñas } \\
\text { Tasa neta de asistencia a la } \\
\text { escuela secundaria } \\
\text { (ajustada)* }\end{array}$ \\
\hline Hodh Echargui & 17.7 & 13.1 \\
Hodh El Gharbi & 15.2 & 18.8 \\
Assaba & 19.7 & 13.3 \\
Gorgol & 21.6 & 18.3 \\
Brakna & 33.8 & 29.4 \\
Trarza & 32.2 & 36.7 \\
Adrar & 34.3 & 23.8 \\
Nouadhibou & 47.1 & 42.2 \\
Tagant & 29.2 & 25.4 \\
Guidimakha & 21.0 & 10.1 \\
Tiris Zemour & 42.4 & 46.0 \\
Inchiri & 46.8 & 39.2 \\
Nouakchott & 48.4 & 44.9 \\
\hline
\end{tabular}

* Tasa neta de asistencia a la escuela secundaria basada en 25-49 casos no ponderados.

Fuente: elaboración propia con base en MICS 5, 2015.

El nivel terciario incluye los tres mayores hospitales del país, llamados hospitales nacionales, y cinco centros especializados, situados todos ellos en Nouakchott. A esta infraestructura pública se añade la atención médica privada, concentrada mayoritariamente en la capital del país (Figura 5).

Esta estructura no garantiza una completa cobertura sanitaria, pues sólo 69 por ciento de la población tiene acceso a la atención primaria (en un radio de cinco km). Así en wilayas como Inchiri la cobertura es de 53 por ciento y en Hodh el Gharbi y Tagant de 55 y 56 por ciento, respectivamente, mientras que en Nouakchott es de 99 por ciento. Es decir, que la equidad aún no se ha tenido en cuenta de forma sistemática en los Planes Nacionales de Desarrollo Sanitario del país (PNDS, 2017). 


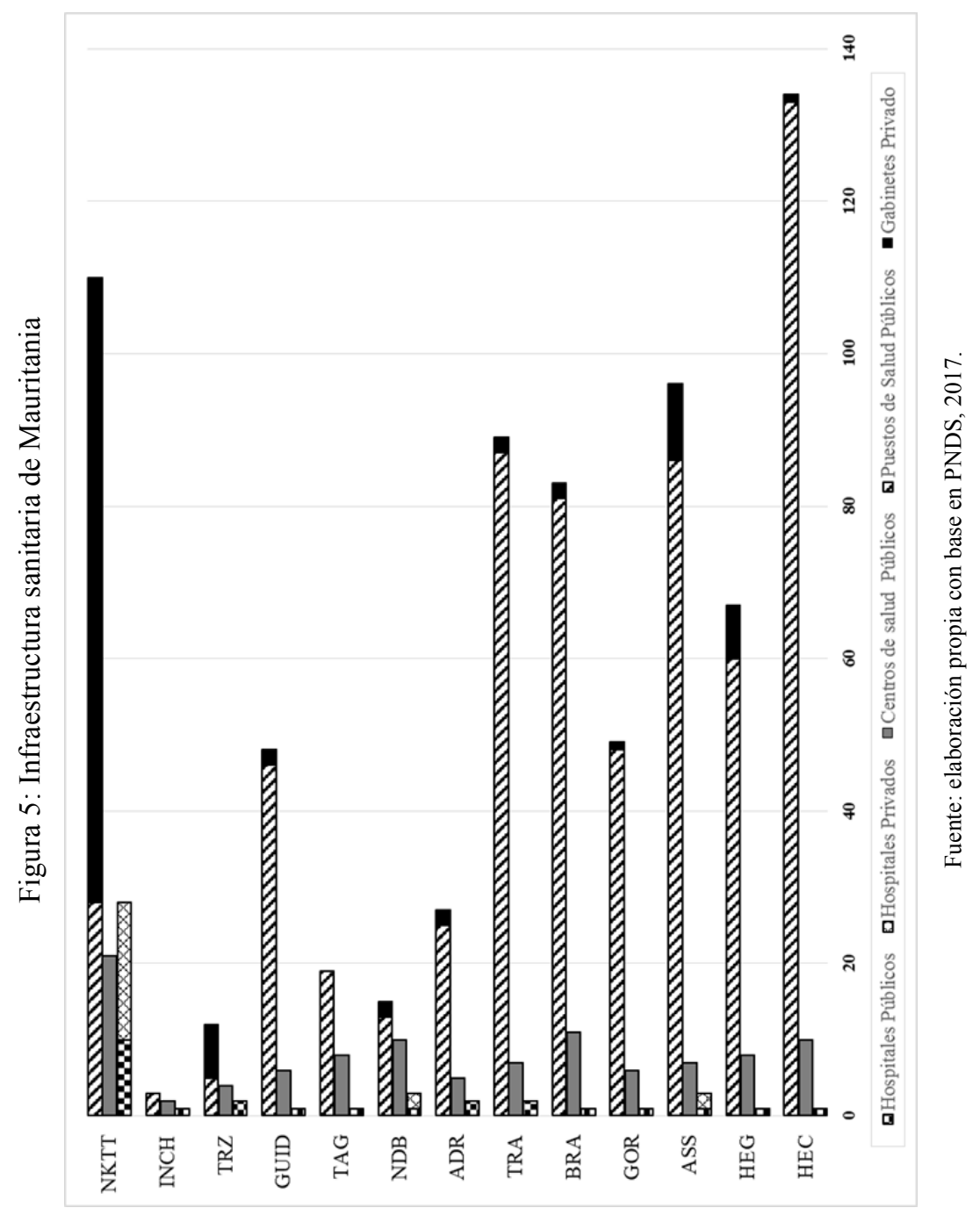


En consecuencia, siguen existiendo disparidades en la prestación de servicios de salud entre las zonas urbanas y rurales, entre las wilayas más ricas y las más pobres, y entre las zonas geográficas según el acceso terrestre, ya sea por falta de vías de comunicación o porque pueden quedar episódicamente incomunicadas por inundaciones. A estos factores se añade la falta de disponibilidad de personal sanitario o el mal estado técnico de muchas de las infraestructuras.

Lo anteriormente expuesto implica un gran riesgo para la calidad de vida de una gran parte de la población y, en especial, para las jóvenes, máxime cuando se quedan embarazadas a temprana edad. La falta de atención sanitaria en este tipo de embarazos y partos conlleva graves problemas para su salud y la de sus descendientes. Como señala la pediatra Haidy Haimoudane: "las adolescentes se ven afectadas por problemas de orden psicológico, como la depresión puerperal, y por otros de orden fisiológico como las fístulas, la ruptura del útero, distinto tipo de infecciones, etcétera." En consecuencia, los partos atendidos por personal cualificado se convierten en una adecuada herramienta para medir el nivel de atención sanitaria a los embarazos tempranos. Un análisis de correlación entre los partos atendidos y la cobertura sanitaria por wilayas evidencia la relación positiva y significativa entre estas dos variables $\left(R=0.68^{*} ; \mathrm{p}<0.05\right)$.

\section{Discusión de RESULTADOS}

Como ya hemos señalado en el apartado de metodología, los distintos parámetros analizados en este estudio fueron correlacionados, de forma que se logra demostrar que la alfabetización y escolarización secundaria tienen una relación inversa con la fecundidad, es decir, que a mayores niveles de alfabetización y de escolarización secundaria existe una menor propensión a tener hijos desde temprana edad, y viceversa. Las pruebas de correlación, sin embargo, no implican causalidad, pudiendo afectar en las dos direcciones. Es decir, la tendencia de tener hijos a temprana edad puede dificultar la educación de la madre, a lo que también apunta el testimonio de Haidy Haimoudane: "la sensibilización sobre las cuestiones relativas a los embarazos precoces facilita el acceso a la educación" y, en sentido complementario, la menor educación puede favorecer el tener hijos a temprana edad. También se demuestra que la incidencia de la pobreza tiene una correlación directa con los altos índices de fecundidad y que hay una correlación inversa entre los partos atendidos por personal cualificado y la fecundidad temprana. 
Sin embargo, en el proceso de elaboración del clúster se probó, a través del análisis de varianza no paramétrico Krukall Wallis (prueba de análisis de varianzas para variables discretas), que la variable del nivel de escolaridad secundaria de las mujeres no tenía incidencia en la formación de los grupos de wilayas, para el caso de mujeres de 15-19 años que comienzan su vida fértil, a diferencia de las demás variables analizadas. En síntesis, los indicadores utilizados revelan los resultados que se ofrecen en la Tabla 5 y la Figura 6.

Tabla 5: Correlaciones para el análisis clúster

\begin{tabular}{|c|c|c|c|c|c|}
\hline & $\begin{array}{c}\text { Tasa } \\
\text { alfabetización } \\
>15 \text { años }\end{array}$ & $\begin{array}{c}\text { Tasa neta } \\
\text { escolarización } \\
\text { nivel secundario } \\
\text { (15-24 años) }\end{array}$ & $\begin{array}{l}\text { Población } \\
\text { rural (\%) }\end{array}$ & $\begin{array}{l}\text { Inciden- } \\
\text { cia de la } \\
\text { pobreza } \\
(\%)\end{array}$ & $\begin{array}{c}\text { Parto atendido } \\
\text { por personal } \\
\text { cualificado } \\
(\%)\end{array}$ \\
\hline $\begin{array}{l}\text { Mujeres que } \\
\text { comienzan su vida } \\
\text { fértil antes de los } \\
15(\%)\end{array}$ & $-753 * *$ & $-659 *$ & 544 & $-560^{*}$ & $-632 *$ \\
\hline $\begin{array}{l}\text { Mujeres de } 15-19 \\
\text { que comienzan su } \\
\text { vida fértil }(\%)\end{array}$ & $-753 * *$ & -500 & 330 & $-599 *$ & $-648^{*}$ \\
\hline $\begin{array}{l}\text { Mujeres de } 20-24 \\
\text { que comenzaron su } \\
\text { vida fértil antes de } \\
\text { los } 19(\%)\end{array}$ & $-907 * *$ & $-769 * *$ & 516 & $-742 * *$ & $-703 * *$ \\
\hline
\end{tabular}

*Correlation is significant at the 0.05 level (2-tailed) **Correlation significant at the 0.01 level (2-tailed).

Fuente: elaboración propia con base enRecensement Général de la Population et de l'Habitat, 2013, EPCV, 2014 y MICS 5, 2015.

Desde una perspectiva geográfica, este análisis multivariante nos permitió establecer cuatro grupos de entidades administrativas: i) La wilaya de Gorgol, con los menores niveles de ingreso del país y con menor número de proyectos de intervención sanitaria. Más de un cuarto de sus adolescentes comenzaron su vida fértil entre los 15 y 19 años. ii) Wilayas más pobres, con mayor proporción de población rural y/o nómada y con menor nivel de alfabetización por parte de su población femenina (Adrar,Trarza, Tagant, Brakna, Guidimakha, Assaba, Hodh Gharby y Hodh Chargui). iii) La wilaya de Inchiri, que se diferencia de las demás porque tiene muy poca población y un predominio de hombres entre los 15-59 años (190 h/100 $\mathrm{m}$ ), que han llegado a ella atraídos por el trabajo minero. iv) wilayas con un mayor nivel de urbanización, con una mayor renta y con una mayor proporción de mujeres alfabetas (Tiris Zemmour, Nouadhibou y Nouakchott). 
La maternidad a edades tempranas en Mauritania: una perspectiva geodemográfica / J. DOMINGUEZ MUJICA et al.

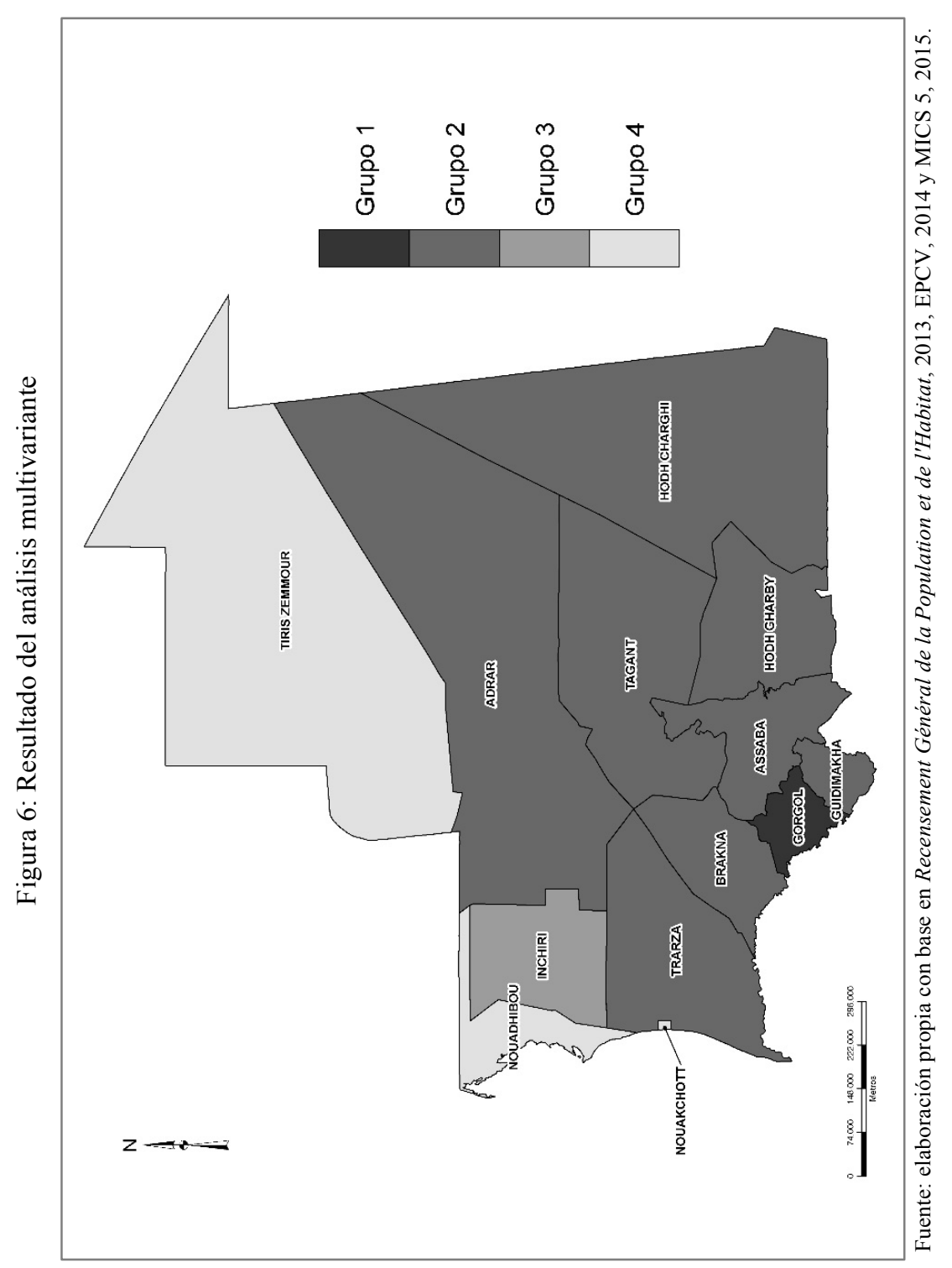


Cada una de estas categorías puede orientar los programas que se han de desarrollar para definir las actuaciones en materia de prevención de embarazos de adolescente en Mauritania.

\section{CONCLusiones}

En una recapitulación de la investigación desarrollada se pone de manifiesto la importancia que guardan los embarazos tempranos en Mauritania con la pobreza, la ruralidad, el nomadismo, las creencias religiosas y ciertas prácticas que descansan en tradiciones y costumbres difíciles de erradicar. Al mismo tiempo se demuestra que un mayor nivel de alfabetización femenino y una mayor atención médica y sanitaria contribuyen a la disminución de los embarazos tempranos. Ahora bien, estos indicadores no se pueden abstraer del contexto geográfico, pues se aprecian profundas diferencias en su distribución territorial, de forma que se hace necesario reconocer los factores que determinan el reparto espacial de la población y la riqueza cuando se diseñan los programas para erradicar o disminuir la incidencia de los embarazos a temprana edad.

Por último, las entrevistas en profundidad realizadas a personas cualificadas en la defensa de los derechos de las mujeres y que velan por su salud reproductiva aportan algunas ideas y recomendaciones que demuestran lo que se está llevando a cabo para hacer más efectivos los protocolos de actuación y que se sintetizan a continuación como conclusiones del estudio.

El cambio de las normas sociales, especialmente las prácticas culturales como la mutilación genital femenina y el matrimonio infantil, es un proceso muy largo, por lo que se desarrollan programas de comunicación que pretenden crear conciencia en las comunidades locales a partir de distintas acciones como: apoyar la resiliencia de las familias, especialmente en las zonas rurales, para promover la educación, y la de las niñas en particular; apoyar el empoderamiento de las mujeres con un fuerte enfoque que mantenga a las niñas en la escuela; realizar una campaña de cambio de comportamiento y mentalidad para los padres que, generalmente, son los únicos que deciden sobre el matrimonio de sus hijas; sensibilizar sobre las malas consecuencias de esta práctica en la salud y establecer un diálogo inclusivo sobre este tema.

A estas consideraciones se suman algunas otras propuestas, propias del ámbito específico de la salud, como las de poner en marcha programas de información sobre salud reproductiva y sexualidad, dirigidos especialmente a los adolescentes, así como el facilitarles el acceso a los medios anticonceptivos. 
La maternidad a edades tempranas en Mauritania: una perspectiva geodemográfica / J. DOMÍNGUEZ MUJICA et al.

\section{REFERENCIAS BIBLIOGRÁFICAS}

Acharya, D. R., Bhattarai, R., Poobalan, A., Teijlingen, V. E., y Chapman, G., 2014, "Factors associated with teenage pregnancy in South Asia", en Health Science Journal, 4(1), pp. 3-14.

Agencia Francaise de Developpement (AFD), 2016, Panorama 2016 - AFD Annual Report https://www.afd.fr/en/panorama-2016-afd-group-annual-report (consulta 18 de agosto de 2019).

Alarcón Argota, R., Coello Larrea, J., Cabrera García, J., and Monier Despeine, G., 2009, "Factores que influyen en el embarazo en la adolescencia", en Revista Cubana de Enfermería, 25(1-2), pp.1-14.

Almanza, C. H. y Sahn, D. E., 2018, "Early childbearing, school attainment, and cognitive skills: evidence from Madagascar", en Demography, 55(2), pp. 643-668.

Amin, S. y Bajracharya, A., 2011, "Costs of marriage- -marriage transactions in the developing world", en Popline https://www.popline.org/node/644421 (consulta 18 de agosto de 2019).

Associated Press, 2007, Mauritania struggles with love of fat women Government trying to change desert culture that force-feeds girls, http://www.nbcnews.com/ $\mathrm{id} / 18141550 /$ ns/health-health_care/t/mauritania-struggles-love-fat-women/\#.

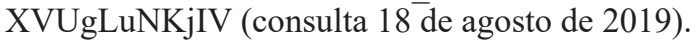

Barcelos, C. A., 2014, "Producing (potentially) pregnant teen bodies: Biopower and adolescent pregnancy in the USA", in Critical Public Health, 24(4), pp. 476488. DOI: $10.1080 / 09581596.2013 .853869$

Binstock, G., y Näslund-Hadley, E., 2013, "Maternidad adolescente y su impacto sobre las trayectorias educativas y laborales de mujeres de sectores populares urbanos de Paraguay", en Papeles de población, 19(78), pp. 15-40.

Calvès, A. E., 2000, "Premarital childbearing in urban Cameroon: paternal recognition, child care and financial support", in Journal of comparative family studies, 31(4), pp. 443-461. DOI: 10.3138/jcfs.31.4.443

Centro Latinoamericano y Caribeño de Demografía (CELADE), 2004, La fecundidad en América Latina: ¿Transición o revolución? https://repositorio.cepal.org/ bitstream/handle/11362/6729/S043186_es.pdf (consulta 18 de agosto de 2019).

Commission Nationale des Droits de l'Homme, 2017, Rapport thématique: Droits des femmes en Mauritanie. Juillet. http://www.cndh.mr/images/rapport thematique_de_la_cndh_sur_les_droits_des_femme_2017.pdf (consulta 18 de ăgosto de 2019).

Chackiel, J., 2004, "La transición de la fecundidad en América Latina 1950-2000”, en Papeles de población, 10(41), pp. 9-58.

Chandra-Mouli, V., Camacho, A. V., y Michaud, P. A., 2013, “WHO guidelines on preventing early pregnancy and poor reproductive outcomes among adolescents in developing countries", en Journal of adolescent health, 52(5), pp. 517-522. DOI: 10.1016/j.jadohealth.2013.03.002 
El País, 2019, Niñas forzadas a comer para casarlas. https://elpais.com/elpais/2018/11/07/planeta_futuro/1541597795_682605.html (consulta 18 de agosto de 2019).

Ford Foundation (Girls not Brides), 2013, Mapping child marriage in West Africa. New Ford Foundation Study. https://www.girlsnotbrides.es/mapping-child-marriage-in-west-africa-new-ford-foundation-study/?view_original (consulta 18 de agosto de 2019).

Ganchimeg, T., Ota, E., Morisaki, N., Laopaiboon, M., Lumbiganon, P., Zhang, J., Yamdamsuren, B., Temmerman, M., Say, L., Tuncalp, O., Vogel, J.P., Souza, J.P., Mori, R., 2014, "Pregnancy and childbirth outcomes among adolescent mothers: a World Health Organization multicountry study", en An International Journal of Obstetrics and Ginaecology, 121, pp. 40-48. DOI: 10.1111/1471-0528.12630

Gausman, J., Langer, A., Austin, S. B. y Subramanian, S. V., 2019, “Contextual variation in early adolescent childbearing: a multilevel study from 33,822 communities in 44 low-and middle-income countries", en Journal of Adolescent Health, 64(6), pp. 737-745. DOI: 10.1016/j.jadohealth.2018.11.018

Goody, J., 1990, The Oriental, the Ancient and the Primitive: Systems of Marriage and the Family in the Pre-industrial Societies of Eurasia. Cambridge University Press.

Green, K. E. and Smith, D. E., 2007, "Change and continuity: childbirth and parenting across three generations of women in the United Arab Emirates", en Child: care, health and development, 33(3), pp. 266-274. DOI:10.1111/j.13652214.2006.00667.x

Groupe de la Banque Africaine de Developpment, 2015, Autonomiser les femmes africaines: Plan d'Action. https://www.afdb.org/fileadmin/uploads/afdb/Documents/Publications/African_Gender_Equality_Index_2015-FR.pdf (consulta 18 de agosto de 2019).

Hindin, M. J., 2014, “Adolescent childbearing and women's attitudes towards wife beating in 25 sub-Saharan African countries", en Maternal and child health journal, 18(6), pp. 1488-1495.

Hof, N., 2015, Joost de Raeymaeker a photographié le gavage des femmes en Mauritanie. http://www.oai13.com/focus/societe/joost-de-raeymaeker-a-photographie-le-gavage-des-femmes-en-mauritanie/ (consulta 18 de agosto de 2019,

Kennedy, E., Gray, N., Azzopardi, P., and Creati, M., 2011, “Adolescent fertility and family planning in East Asia and the Pacific: a review of DHS reports", en Reproductive Health, 8(1), pp. 1-11.

Lemon, E., Hennink, M., y Can Saquic, N. A., 2017, "Pathways to adolescent childbearing among Kaqchikel women in Guatemala". Culture, Health \& Sexuality, 19(10), pp. 1149-1164. DOI: 10.1080/13691058.2017.1298841

León, P., Minassian, M., Borgoño, R., y Bustamante, F., 2008, “Embarazo adolescente", en Revista Pediátrica Electrónica 5(1), pp. 42-51. 
Lloyd, C. B., y Mensch, B. S., 2008, "Marriage and childbirth as factors in dropping out from school: an analysis of DHS data from sub-Saharan Africa", en Population Studies, 62(1), pp. 1-13. DOI: 10.1080/00324720701810840

Lundström, K. E., y Andersson, G., 2012, "Labor-market status, migrant status and first childbearing in Sweden", en Demographic Research, 27, pp. 719-742. DOI: 10.4054/DemRes.2012.27.25

Maswikwa, B., Richter, L., Kaufman, J., y Nandi, A., 2015, "Minimum marriage age laws and the prevalence of child marriage and adolescent birth: evidence from sub-Saharan Africa", en International Perspectives on Sexual and Reproductive Health, 41(2), pp. 58-68. DOI: 10.1363/4105815

Mendoza, W., y Subiría, G., 2013, “El embarazo adolescente en el Perú: situación actual e implicancias para las políticas públicas". Revista peruana de medicina experimental y salud pública, 30, pp. 471-479.

Menkes, C., y Suárez, L., 2003, “Sexualidad y embarazo adolescente en México", en Papeles de población, 9(35), pp. 233-262.

Ministerio de Asuntos Sociales de Mauritania y World Vision, 2018, La Mauritanie engagée à bannir le mariage des enfants http://aidara.mondoblog.org/2018/10/12/ mauritanie-engagee-a-bannir-mariage-enfants/ (consulta 18 de agosto de 2019).

Ministère de la Santé. RIM, 2014, Carte Sanitaire de la Mauritanie. AECID y Misnistère de la Santé, RIM. Disponible en: http://www.sante.gov.mr/?wpfb_ $\mathrm{dl}=99$ (consulta 18 de agosto de 2019).

Ministère de la Santé. RIM, 2017, Plan National de Développement Sanitaire (PNDS) 2017-2020. http://www.sante.gov.mr/?wpfb_dl=205 (consulta $18 \mathrm{de}$ agosto de 2019).

Moghadam, V. M., 2004, "Patriarchy in transition: Women and the changing family in the Middle East", en Journal of Comparative Family Studies, 35(2), pp. 137-162. DOI: $10.3138 /$ jefs.35.2.137

Nahar, Q., y Min, H., 2008, “Trends and determinants of adolescent childbearing in Bangladesh", en Popline https://www.popline.org/node/209752 (consulta 18 de agosto de 2019).

Neal, S., Matthews, Z., Frost, M., Fogstad, H., Camacho, A. V., y Laski, L., 2012, "Childbearing in adolescents aged 12-15 years in low resource countries: a neglected issue. New estimates from demographic and household surveys in 42 countries", en Acta obstetricia et gynecologica Scandinavica, 91(9), pp. 11141118. DOI: 10.1111/j.1600-0412.2012.01467.x

Office National des Statistiques (ONS), 2016, Rapport de l'Etude du Genre à partir des données du RGPH. http://www.ons.mr/index.php/ons_(consulta 18 de agosto de 2019).

Office National des Statistiques (ONS), 2014, Enquête Permanente sur les Conditions de Vie des ménages 2014. http://www.ons.mr/images/Archive/images/EPCV/ PROFIL\%20DE\%20LA\%20PAUVRETE\%20EN\%20MAURITANIE\%202014. pdf (consulta 18 de agosto de 2019). 
Office National des Statistiques (ONS), 2013, Recensement Général de la Population et de l'Habitat (RGPH). http://www.ons.mr/index.php/publications/operations-statistiques/16-rgph-2013 (consulta 18 de agosto de 2019).

Organización Mundial de la Salud (OMS), 2018, El embarazo en la adolescencia, en https://www.who.int/es/news-room/fact-sheets/detail/adolescent-pregnancy (consulta 18 de agosto de 2019).

Penman-Aguilar, A., Carter, M., Snead, M. C., y Kourtis, A. P., 2013, "Socioeconomic disadvantage as a social determinant of teen childbearing in the US", en Public Health Reports, 128(2_suppl1), 5-22.

Rguibi, M., y Belahsen, R., 2006, "Fattening practices among Moroccan Saharawi women”, en Eastern Mediterranean Health Journal, 12 (5), pp. 619-624.

Santelli, J. S., Song, X., Garbers, S., Sharma, V., y Viner, R. M., 2017, “Global trends in adolescent fertility, 1990-2012, in relation to national wealth, income inequalities, and educational expenditures", en Journal of Adolescent Health, 60(2), pp. 161-168. DOI: 10.1016/j.jadohealth.2016.08.026

Santhya, K.G., 2011, "Early marriage and sexual and reproductive health vulnerabilities of young women: a synthesis of recent evidence from developing countries", en Current Opinion in Obstetrics and Gynecology, 23(5), pp. 334-339. DOI: $10.1097 / \mathrm{GCO} .0 \mathrm{~b} 013 \mathrm{e} 32834 \mathrm{a} 93 \mathrm{~d} 2$

Shakya, H. B., Weeks, J. R., y Christakis, N. A., 2019, Do village-level normative and network factors help explain spatial variability in adolescent childbearing in rural Honduras?, SSM-Population Health, 100371. DOI: 10.1016/j.ssmph.2019.100371. In press.

Singh, S., y Darroch, J. E., 2000, “Adolescent pregnancy and childbearing: levels and trends in developed countries", en Family planning perspectives, 32(1), pp.14-23.

Singh, S., 1998, “Adolescent Childbearing in Developing Countries: A Global Review”, en Studies in Family Planning, 29 (2), pp. 117-136.

Sobotka, T., 2008, "The diverse faces of the second demographic transition in Europe", en Demographic research, 19(8), 171-224. DOI: 10.4054/ DemRes.2008.19.8

Stern, C., 2004, "Vulnerabilidad social y embarazo adolescente en México" en Papeles de población, 10(39), pp. 129-158.

Svanemyr, J., Chandra-Mouli, V., Raj, A., Travers, E., y Sundaram, L., 2015, "Research priorities on ending child marriage and supporting married girls", en Reproductive Health, 12(1), pp. 1-4. DOI 10.1186/s12978-015-0060-5

Tertilt, M., 2005, "Polygyny, fertility, and savings", en Journal of Political Economy, 113(6), pp. 1341-1371. DOI: 10.1086/498049

Thaithae, S. y Thato, R., 2011, "Obstetric and perinatal outcomes of teenage pregnancies in Thailand", en Journal of pediatric and adolescent gynecology, 24(6), pp. 342-346. DOI: 10.1016/j.jpag.2011.02.009 
UNFPA, 2016, Sahel Women's Empowerment and Demographic Dividend (SWEDD), en https://wcaro.unfpa.org/sites/default/files/pub-pdf/SWEDD_ENG. pdf (consulta 18 de agosto de 2019).

UNICEF, 2015, Mauritanie. Enquête par grappes à indicateurs multiples MICS5 2015 Rapport final. http://www.ons.mr/images/mics/MICS5_rapport.pdf (consulta 18 de agosto de 2019).

UNICEF, 2011, Mauritanie. Suivi de la situation des femmes et des enfants Enquête par Grappes à Indicateurs Multiples 2011. Raport final MICS4. https://micssurveys-prod.s3.amazonaws.com/MICS4/West\%20and\%20Central\%20Africa/ Mauritania/2011/Final/Mauritania\%202011\%20MICS_French.pdf (consulta 18 de agosto de 2019).

Wahhaj, Z., 2015, "A theory of child marriage". School of Economics Discussion Papers No. 1520. University of Kent, School of Economics, Canterbury.

Walker, J. A., 2012, "Early marriage in Africa-trends, harmful effects and interventions", en African Journal of Reproductive Health, 16(2), pp. 231-240.

Were, M., 2007, "Determinants of teenage pregnancies: The case of Busia District in Kenya", en Economics and Human Biology, 5(2), pp. 322-339. DOI: 10.1016/j. ehb.2007.03.005

Wodon, Q., Malé, C., y Onagoruwa, A., 2019, “A simple approach to measuring the share of early childbirths likely due to child marriage in developing countries", en Forum for Social Economics (pp. 1-14). Routledge.

\section{RESUMEN CURRICULAR DE LAS AUTORAS}

\section{Josefina Domínguez Mujica}

Doctora en Geografía por la Universidad de Las Palmas de Gran Canaria y Catedrática de Geografía Humana de la misma Universidad. Ha dedicado gran parte de su actividad investigadora a la Geografía de la Población y al estudio de las migraciones. Ha participado en numerosos proyectos de investigación sobre procesos migratorios financiados por instituciones nacionales (Planes Nacionales de $\mathrm{I}+\mathrm{D}+\mathrm{i}$ ) y ha participado en Comités de Expertos en materia de inmigración, habiendo realizado sendos informes para el Gobierno de Canarias, el Parlamento de Canarias, la Comisión Española de Ayuda al Refugiado y la Organización Internacional de Migraciones. Dirige el Grupo de Investigación de la ULPGC de Sociedades y Espacios Atlánticos y, preside la Comisión Global Change and Human Mobility de la Unión Geográfica Internacional. Además, ha publicado un gran número de capítulos y artículos en libros y revistas nacionales e internacionales de gran prestigio editorial.

Dirección electrónica: josefina.dominguezmujica@ulpgc.es

Registro ORCID: http://orcid.org/0000-0001-7460-5553 
Mercedes de los Ángeles Rodríguez Rodríguez

Licenciada en Geografía en Cuba, homologada en España. Máster y Doctora por la Universidad de Las Palmas de Gran Canaria (ULPGC). Trabajo como profesora Ayudante Doctora a tiempo completo en el área de Geografía Humana ULPGC. Mis temas de investigación versan en aspectos de demografía, ambiente y salud, así como en el perfil sociodemográfico de diferentes poblaciones. Consultora en temas demográficos, relacionados con geografía de la salud, y en la aplicación de técnicas cualitativas en los estudios de poblaciones en África, en especial en Mauritania y Guinea Ecuatorial. He participado en proyectos financiados por la Agencia Española de Cooperación Internacional al Desarrollo (AECID) y América Latina, por la Organización Panamericana de la Salud (OPS-OMS).

Dirección electrónica: mercedes.rodriguez@ulpgc.es

Registro ORCID: http://orcid.org/0000-0003-1015-2027

\section{Laudy Rivero Rodríguez}

Licenciada en Medicina y Cirugía, por la Universidad de Las Palmas de Gran Canaria (2014). Participó en el Programa Erasmus, Universidad La Sapienza (Roma, Italia) 011/12 y posteriormente en el Programa Universitario AMIR, 2015/16: Formación médica para la residencia oficial de médicos internos (MIR) (Barcelona, España). Obtuvo el puesto como médico interno residente de Medicina Familiar y Comunitaria en La Laguna-Tenerife Norte, Servicio Canario de Salud. Estancia de investigación en la Clínica Mayo, en Rochester, Minnesota, Estados Unidos (3 meses). Dentro de sus trabajos de investigación se encuentran los relacionados con la salud de la mujer dentro de la comunidad, y el diseño de estrategias para aumentar su calidad de vida.

Dirección electrónica: laudyrivero@gmail.com 